\title{
A Criterion on Existence and Uniqueness of Behavior in Electric Circuit
}

\author{
Takuya Hirata* , Eko Setiawan ${ }^{*}$, Kazuya Yamaguchi ${ }^{* *}$, and Ichijo Hodaka** \\ *Interdisciplinary Graduate School of Agriculture and Engineering, University of Miyazaki \\ ** Department of Control Engineering, National Institute of Technology Nara College \\ **** Department of Environmental Robotics, Faculty of Engineering, University of Miyazaki
}

\section{Article Info}

\section{Article history:}

Received Feb 5, 2016

Revised May 22, 2016

Accepted Jun 8, 2016

\section{Keyword:}

behavior of electric circuit

switching circuit

circuit analysis

\begin{abstract}
Behavior of electric circuits can be observed by solving circuit equations symbolically as well as numerically. In general, symbolic computation for circuits with certain number of circuit elements needs much more time than numerical computation. It is reasonable to check the existence and uniqueness of the solution to circuit equations beforehand in order to avoid computation for the case of no solution. Indeed, some circuits have no solution; in that case, one should notice it and avoid to wait meaningless computation. This paper proposes a new theorem to check whether given circuit equations have a solution and their voltages and currents of all circuit elements are uniquely determined or not. The theorem is suitable for developing a computer algorithm and helps quick symbolic computation for electric circuits.
\end{abstract}

Copyright (c) 2016 Institute of Advanced Engineering and Science. All rights reserved.

\section{Corresponding Author:}

Ichijo Hodaka

Department of Environmental Robotics, Faculty of Engineering, University of Miyazaki

1-1, Gakuen Kibanadai Nishi, Miyazaki, 889-2192, Japan

hijhodaka@cc.miyazaki-u.ac.jp

\section{INTRODUCTION}

Any simulation of behavior of an actual circuit is based on modelling of the circuit with idealization. The modelling is a crucial step of analysis and design; it depicts the actual circuit as a circuit diagram, determines a working point, and linearizes characteristics of electric components in the actual circuit with a working frequency. Behavior of the actual circuit is represented by a solution to circuit equations derived from the circuit diagram and the fundamental laws such as Kirchhoff's laws, Ohm's law, and the electric characteristics of inductor and capacitor. SPICE[1], a defacto standard circuit simulator, simulates and plots the behavior by numerical calculation. That is, we there assume existence and uniqueness of behavior. We also assume them if we measure behavior of an actual circuit. Hence, a modelling process should not sacrifice existence and uniqueness of behavior; voltages and currents at components in the actual circuit or in the simulation model should be single-valued functions defined on all time.

Some studies address circuit diagrams which have lost existence and uniqueness of behavior. For example, some of inductor currents and capacitor voltages in the circuit diagram are not eligible for a member of state variables[2]-[5]. This can be translated into a problem to find a spanning tree in the circuit by graph theory[3]-[5]. However, little is known about a direct procedure to check a given circuit diagram.

In this paper, we propose a quick criterion to check whether a solution to linear circuit equations exists and is uniquely determined or not. The criterion enables us to validate complicated circuits such as a switching circuit with computers, and thus draw a proper circuit diagram - a diagram which has existence and uniqueness of behavior with help of computers. Notice that the criterion is written in a symbolic equation. That means our result would be a fundamental result to contribute the field of symbolic calculation[6][7] of electric circuits. 


\section{LAWS OF ELECTRIC CIRCUITS AND CIRCUIT EQUATIONS}

For a given circuit diagram, circuit equations are defined by the fundamental laws, Kirchhoff's voltage law(KVL), Kirchhoff's current law(KCL), Ohm's law, and the electric characteristics of inductor and capacitor, where all variables in the laws and the characteristics are assumed to be single-valued functions of time $t$. In general, we have

$$
\begin{aligned}
A w+B y+C z & =0 \\
H\left(x(t)-x\left(t_{0}\right)\right) & =\int_{t_{0}}^{t} z_{1}(p) d p
\end{aligned}
$$

where, $A, B, C$, and $H$ are constant matrices whose entries are determined by the laws and the characteristics of electric circuit and resistance of resistors,

$$
x=\left[\begin{array}{c}
i_{L} \\
v_{C}
\end{array}\right], u=\left[\begin{array}{c}
v_{V} \\
i_{I}
\end{array}\right], w=\left[\begin{array}{c}
u \\
x
\end{array}\right], y: \text { the vector of node voltages, } z_{1}=\left[\begin{array}{c}
v_{L} \\
i_{C}
\end{array}\right], z=\left[\begin{array}{c}
i_{V} \\
v_{I} \\
v_{R} \\
i_{R} \\
z_{1} \\
v_{S} \\
i_{S}
\end{array}\right],
$$

and $v_{V}, v_{I}, v_{C}, v_{L}, v_{R}$, and $v_{S}$ are voltages of voltage sources, current sources, capacitors, inductors, resistors and switches, and $i_{V}, i_{I}, i_{C}, i_{L}, i_{R}$, and $i_{S}$ are currents of voltage sources, current sources, capacitors, inductors, resistors and switches, and $v_{N}$ is potential of nodes, respectively. This paper assumes that all the variables are Riemann integrable, and thus the notation $\int$ in the equation (2) is Riemann integral (for definition of integral, see e.g. [8]).

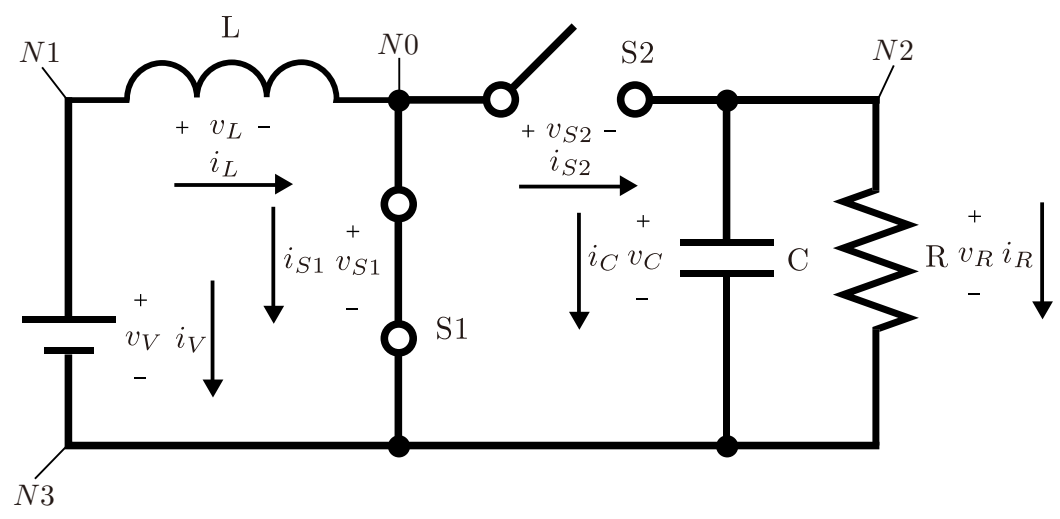

Figure 1. A boost converter - CCM (S1: ON, $S 2$ : OFF)

Example Figure 1 and 2 represent so-called continuous conduction mode (CCM) and discontinuous conduction mode (DCM) of a boost converter [9], respectively. Circuit equations of the circuit diagram in Figure 1 are represented 
in the form (1) and (2), where

$$
A=\left[\begin{array}{ccc}
1 & 0 & 0 \\
0 & 0 & 0 \\
0 & 0 & 1 \\
0 & 0 & 0 \\
0 & 0 & 0 \\
0 & 0 & 0 \\
0 & -1 & 0 \\
0 & 0 & 0 \\
0 & 1 & 0 \\
0 & 0 & 0 \\
0 & 0 & 0 \\
0 & 0 & 0 \\
0 & 0 & 0
\end{array}\right], B=\left[\begin{array}{cccc}
-1 & 1 & 0 & 0 \\
-1 & 0 & 1 & 0 \\
0 & 1 & 0 & -1 \\
0 & 1 & 0 & -1 \\
0 & 1 & -1 & 0 \\
0 & 0 & -1 & 1 \\
0 & 0 & 0 & 0 \\
0 & 0 & 0 & 0 \\
0 & 0 & 0 & 0 \\
0 & 0 & 0 & 0 \\
0 & 0 & 0 & 0 \\
0 & 0 & 0 & 0 \\
0 & 0 & 0 & 0
\end{array}\right], C=\left[\begin{array}{ccccccccc}
0 & 0 & 0 & 0 & 0 & 0 & 0 & 0 & 0 \\
0 & 0 & 0 & 1 & 0 & 0 & 0 & 0 & 0 \\
0 & 0 & 0 & 0 & 0 & 0 & 0 & 0 & 0 \\
0 & 1 & 0 & 0 & 0 & 0 & 0 & 0 & 0 \\
0 & 0 & 0 & 0 & 0 & 1 & 0 & 0 & 0 \\
0 & 0 & 0 & 0 & 0 & 0 & 0 & 1 & 0 \\
-1 & 0 & 0 & 0 & 0 & 0 & 0 & 0 & 0 \\
1 & 0 & 1 & 0 & 1 & 0 & 1 & 0 & 0 \\
0 & 0 & 0 & 0 & 0 & 0 & -1 & 0 & -1 \\
0 & 0 & -1 & 0 & -1 & 0 & 0 & 0 & 1 \\
0 & 1 & -R & 0 & 0 & 0 & 0 & 0 & 0 \\
0 & 0 & 0 & 0 & 0 & 1 & 0 & 0 & 0 \\
0 & 0 & 0 & 0 & 0 & 0 & 0 & 0 & 1
\end{array}\right],
$$

$$
H=\left[\begin{array}{ll}
L & 0 \\
0 & C
\end{array}\right], x=\left[\begin{array}{l}
i_{L} \\
v_{C}
\end{array}\right], z_{1}=\left[\begin{array}{l}
v_{L} \\
i_{C}
\end{array}\right], w=\left[\begin{array}{l}
v_{V} \\
i_{L} \\
v_{C}
\end{array}\right], y=\left[\begin{array}{c}
v_{N 1} \\
v_{N 3} \\
v_{N 0} \\
v_{N 2}
\end{array}\right] \text {, and } z=\left[\begin{array}{c}
i_{V} \\
v_{R} \\
i_{R} \\
v_{L} \\
i_{C} \\
v_{S 1} \\
i_{S 1} \\
v_{S 2} \\
i_{S 2}
\end{array}\right] \text {. }
$$

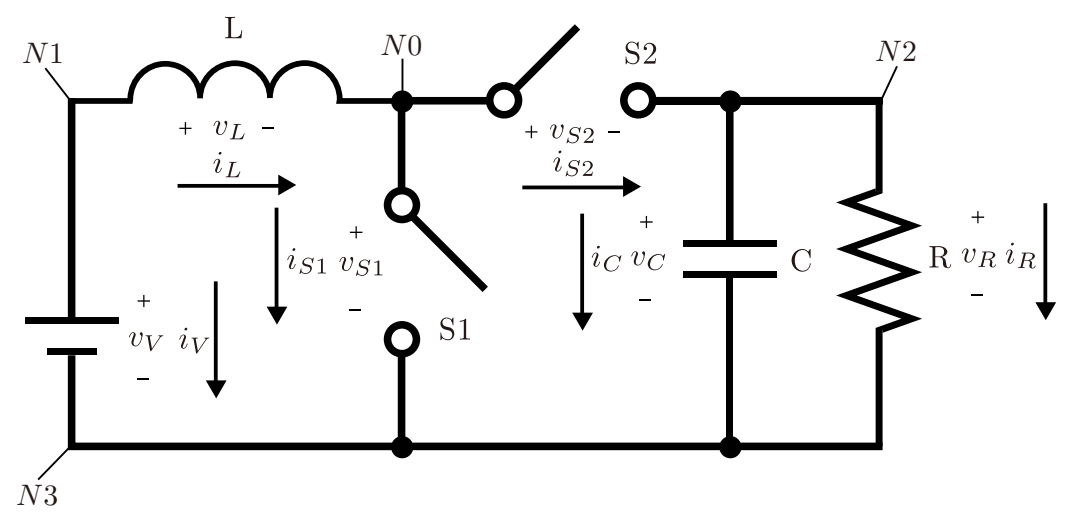

Figure 2. A boost converter - DCM (S1: OFF, $S 2$ : OFF)

Circuit equations of Figure 2 are represented in the form (1) and (2), where

$$
A=\left[\begin{array}{ccc}
1 & 0 & 0 \\
0 & 0 & 0 \\
0 & 0 & 1 \\
0 & 0 & 0 \\
0 & 0 & 0 \\
0 & 0 & 0 \\
0 & -1 & 0 \\
0 & 0 & 0 \\
0 & 1 & 0 \\
0 & 0 & 0 \\
0 & 0 & 0 \\
0 & 0 & 0 \\
0 & 0 & 0
\end{array}\right], B=\left[\begin{array}{cccc}
-1 & 1 & 0 & 0 \\
-1 & 0 & 1 & 0 \\
0 & 1 & 0 & -1 \\
0 & 1 & 0 & -1 \\
0 & 1 & -1 & 0 \\
0 & 0 & -1 & 1 \\
0 & 0 & 0 & 0 \\
0 & 0 & 0 & 0 \\
0 & 0 & 0 & 0 \\
0 & 0 & 0 & 0 \\
0 & 0 & 0 & 0 \\
0 & 0 & 0 & 0 \\
0 & 0 & 0 & 0
\end{array}\right], C=\left[\begin{array}{ccccccccc}
0 & 0 & 0 & 0 & 0 & 0 & 0 & 0 & 0 \\
0 & 0 & 0 & 1 & 0 & 0 & 0 & 0 & 0 \\
0 & 0 & 0 & 0 & 0 & 0 & 0 & 0 & 0 \\
0 & 1 & 0 & 0 & 0 & 0 & 0 & 0 & 0 \\
0 & 0 & 0 & 0 & 0 & 1 & 0 & 0 & 0 \\
0 & 0 & 0 & 0 & 0 & 0 & 0 & 1 & 0 \\
-1 & 0 & 0 & 0 & 0 & 0 & 0 & 0 & 0 \\
1 & 0 & 1 & 0 & 1 & 0 & 1 & 0 & 0 \\
0 & 0 & 0 & 0 & 0 & 0 & -1 & 0 & -1 \\
0 & 0 & -1 & 0 & -1 & 0 & 0 & 0 & 1 \\
0 & 1 & -R & 0 & 0 & 0 & 0 & 0 & 0 \\
0 & 0 & 0 & 0 & 0 & 0 & 1 & 0 & 0 \\
0 & 0 & 0 & 0 & 0 & 0 & 0 & 0 & 1
\end{array}\right]
$$


and the other notations are already defined as (5).

The variable $v_{L}$ cannot be uniquely determined on the algebraic circuit equations (6) of Figure 2 for any $v_{V}, i_{L}$, and $v_{C}$, which is understood by looking at the circuit diagram. We will propose a theorem to capture the above situation by rank calculations of matrices $A, B$ and $C$ in the algebraic equation (1).

\section{THE EXISTENCE AND UNIQUENESS OF SOLUTION OF ALGEBRAIC CIRCUIT EQUATIONS}

If for any $w$, a solution $(y, z)$ to the algebraic circuit equation (1) of a circuit exists and $z$ is unique, then the circuit is said to be proper in this paper. A circuit is said to be improper if it is not proper.

Theorem 1. A circuit is proper if and only if the coefficient matrices of its algebraic equation in the form (1) satisfy

$$
\operatorname{rank}[A B C]=\operatorname{rank}[B C]=\operatorname{rank} B+m \text {. }
$$

where $m$ is the number of column of the matrix $C$.

The proof of Theorem 1 is given in the appendix A. Rank calculation in Theorem 1 is performed on general purpose symbolic computation system widely used. This helps us check whether a circuit is proper or not, even if it is large and complicated, and then difficult to check by hand.

Example (A proper circuit) We recall the circuit in Figure 1 and obtain

$$
\operatorname{rank}[A B C]=12, \operatorname{rank}[B C]=12, \operatorname{rank} B=3, m=9
$$

for (4). Therefore the circuit is proper by Theorem 1.

Example (An improper circuit) We recall the circuit in Figure 2 and obtain

$$
\operatorname{rank}[A B C]=12, \operatorname{rank}[B C]=11, \operatorname{rank} B=3, m=9
$$

for (6). Therefore the circuit is improper by Theorem 1.

The whole behavior of a proper circuit is given by Corollary 1 in the appendix A.

\section{CONCLUSION}

Behavior of an actual electric circuit, a set of voltages and currents of all circuit components, is assumed to be expressed as a single-valued function defined on all time, in general. Designers depict the actual circuit as a schematic diagram to simulate behavior of the actual circuit. Careful designers model the circuit as a diagram with a decision of including parasitic elements or not and reflect a working point and a working frequency of the circuit on the diagram. They write circuit equations equivalent to the diagram, and then, solve the equations to simulate behavior of the actual circuit. However, existence and uniqueness which are assumed to behavior of the actual circuit are not necessarily inherited to the solution because the diagram and the equations are a reduction of the actual circuit. This paper has proposed a criterion for the existence and uniqueness to be guaranteed. The proposed criterion is expressed as equality between ranks of coefficient matrices in the circuit equations. Effective and quick calculation of matrix rank is available in general purpose symbolic computing tools. Therefore our result contributes to the field of symbolic computation of electric circuits.

\section{A LEMMA, COROLLARY AND PROOF}

Corollary 1. If a circuit is proper, the equations (1) and (2) are uniquely solved as:

$$
\begin{aligned}
z & =K_{1} x+K_{2} u \\
x(t) & =e^{F\left(t-t_{0}\right)} x\left(t_{0}\right)+e^{F t} \int_{t_{0}}^{t} e^{-F p} G u(p) d p .
\end{aligned}
$$

Proof 1. We immediately obtain the expression (7) by Theorem 1 . This includes $z_{1}=K_{11} x+K_{21} u$. Notice that the matrix $H$ is block-diagonal whose blocks have capacitances $C$ 's and self or mutual inductances ( $L$ 's and $M$ 's respectively) in their entries and is invertible. If we put $F=H^{-1} K_{11}$ and $G=H^{-1} K_{21}$ and apply integration by parts[8] with a matrix exponential function, we have (8).

We remark that the solution (8) is obtained without assuming $x(t)$ to be differentiable. 
Lemma 1. Let $B$ and $C$ be matrices with a common number of rows. The equation $B y+C z=0$ has a solution $z$ for any $y$ if and only if $\operatorname{rank}[B C]=\operatorname{rank} C$.

\section{Proof 2.}

(only if) There is a matrix $Z$ such that $B=C Z$. Then $[B C]=[C Z C]=C\left[Z U_{m}\right]$, where $U_{m}$ is an identity matrix. rank $[B C]=\operatorname{rank} C$, because $\left[Z U_{m}\right]$ is row full rank.

(if) Since rank $[B C]=\operatorname{rank} C$ and $\operatorname{Im}[B C] \supset \operatorname{Im} C, \operatorname{Im}[B C]=\operatorname{Im} C$. Hence $\operatorname{Im} B \subset \operatorname{Im}[B C]=\operatorname{Im} C$. This means that for every fixed $y$, there is a $z$ such that $B y+C z=0$.

Proof 3 (For Theorem 1).

(only if) By the assumption and Lemma $1, \operatorname{rank}[A B C]=\operatorname{rank}[B C]$. Let $w=0$. The solution space $Q$ of the equation (1) is $Q=\operatorname{ker}[B C]$. Let $\left[\begin{array}{l}y_{1} \\ z_{1}\end{array}\right] \in Q$ and $y_{2} \in \operatorname{ker} B$. Since $\left[\begin{array}{c}y_{2} \\ 0\end{array}\right] \in Q, z_{1}=0$. Hence, $\operatorname{dim}(\operatorname{ker}[B C])=$ $\operatorname{dim}(\operatorname{ker} B)$. By rank-nullity theorem, we obtain

$$
\operatorname{rank}[B C]=\operatorname{rank} B+m \text {. }
$$

(if) Let $Q(w)=\left\{\left[\begin{array}{l}y \\ z\end{array}\right] \mid A w+B y+C z=0\right\}$. By Lemma 1, for every fixed $w, Q(w) \neq \phi$. Since rank $[B C] \leq \operatorname{rank} B+$ rank $C$ and rank $C \leq m$ in general, rank $[B C]=\operatorname{rank} B+m$ follows $m=\operatorname{rank}[B C]-\operatorname{rank} B \leq \operatorname{rank} C \leq m$. So,

$$
\operatorname{rank} C=m=\operatorname{rank}[B C]-\operatorname{rank} B .
$$

Use a general equality $\operatorname{rank}[B C]=\operatorname{rank} B+\operatorname{rank} C-\operatorname{dim}(\operatorname{Im} B \cap \operatorname{Im} C)[10]$ and (9) show

$$
\operatorname{dim}(\operatorname{Im} B \cap \operatorname{Im} C)=0 .
$$

Let $\left[\begin{array}{l}y_{1} \\ z_{1}\end{array}\right]$ and $\left[\begin{array}{l}y_{2} \\ z_{2}\end{array}\right] \in Q(w)$. Then $B y_{1}+C z_{1}=B y_{2}+C z_{2}$. By (10), $C\left(z_{1}-z_{2}\right)=0$. The first equality in (9) implies $z_{1}=z_{2}$.

\section{REFERENCES}

[1] L. W. Nagel and D. O. Pederson, SPICE (Simulation Program with Integrated Circuit Emphasis), Memorandum No. ERLM382 Electronic Research Laboratory, 1973.

[2] T. Bashkow, "The A Matrix, New Network Description," IRE Transactions on Circuit Theory, Vol. 4, No. 3, pp. 117-119, 1957.

[3] E. S. Kuh and R.A. Rohrer, "The State-Variable Approach to Network Analysis," Proceedings of the IEEE, Vol. 53, No. 7, pp. 672-686, 1965.

[4] R. A. Rohrer, Circuit Theory: An Introduction to the State Variable Approach, McGraw-Hill, 1972.

[5] D. A. Calahan, Computer-Aided Network Design, Revised Edition, McGraw-Hill, 1972.

[6] A. Luchetta, S. Manetti, and A. Reatti, "SAPWIN-a symbolic simulator as a support in electrical engineering education," IEEE Transactions on Education, Vol. 44, No. 2, 2001.

[7] T. Hirata, K. Yamaguchi, and I. Hodaka, "A Symbolic Equation Modeler for Electric Circuits," ACM Communications in Computer Algebra, Vol. 49, No. 3, Issue 193, 2015.

[8] K. Knopp, Theory and Application of Infinite Series, Blackie, 1951.

[9] R. W. Erickson and D. Maksimović, Fundamentals of Power Electronics, Second Edition, Kluwer, 2004.

[10] P. Lancaster and M. Tismenetsky, The Theory of Matrices, Second Edition, Academic Press, 1985. 